\title{
Research on Inspection Robot for 35KV Small Substation
}

\author{
Zhao Lujia $^{a^{*}}$, Zhang Wenjian ${ }^{\mathrm{b}}$,Zhao Ailin ${ }^{\mathrm{c}}$,Tian Sijia $^{\mathrm{d}}$ \\ Department of Mechanical Engineering, North China Electric Power University, Baoding 071003 \\ China \\ a597837757@qq.com, ${ }^{b}$ zhangwenjiancn@163.com, ${ }^{c} 1473458020 @ q q . c o m$, \\ d1360727243@qq.com
}

Keywords: inspection robot; small substation; Internet monitoring; automatic charging Abstract. For small 35KV unmanned substations' actual need, this paper proposes a scheme about inspection robot for small substations. The scheme realizes the remote inspection robot motion control and the monitor of the substation equipment's appearance and temperature through the client monitoring software and Internet communications. The inspection robot navigates by the magnetic track and positioning himself through the radio frequency identification technology. The inspection robot obtains an external view and infrared thermal image of substation equipment by carrying the HD cameras and infrared thermometer. Finally, the design of a charging mechanism achieves the automatic charging function of inspection robot. The practical results show that the inspection robot in small $35 \mathrm{KV}$ substation runs stable. People can observe substation equipment remotely at the power company and discover timely the abnormal appearance and temperature of substation equipment. The scheme reduces effectively the labor intensity and improves work efficiency.

\section{Introduction}

With the development of technologies and the deepening of the reformation in electric power system, automation level of electric power system has been greatly improved. As a consequence, the inspection of substations tends to flexible manpower line and even unmanned [1]. However, in order to ensure the reliability of power supply, inspection personnel still need to travel long distance to substations and conduct on-site inspections regularly. The operating data of electrical equipments are collected by devices like hand-held infrared thermometer and hand-held computers, etc [2]. Currently, most of the $35 \mathrm{KV}$ substations have achieved unattended. But there are a great number of $35 \mathrm{KV}$ substations scattered locate in the vast territory, which brings inconvenience in inspection. Manual inspection has shortcomings such as labor-intensive, low efficiency, testing quality decentralized and high costs in management [3-4].

Substation inspection robot is one of the best solutions to solve these problems. But currently, inspection robots are mostly used in large substations with guards such as 500KV substations. The inspection robot's body and monitor devices are all located in the substation [5], and its main purpose is to do daily patrol of substations without staff, achieving automation of routine inspection work. The inspection task of $35 \mathrm{KV}$ substation is relatively light because of its small scale. But transport inspection personnel need to drive or walk a long distance to go to the scene to conduct a plurality of unmanned substation inspection. Therefore, the working strength of manual inspection is mainly in travel. Facing this situation, $35 \mathrm{KV}$ substations need a inspection robot system which can place the monitor in power company so that inspection personnel don't have to go to the scene to complete a routine inspection of the substation. This paper presents an inspection robot system scheme according to the actual situation and the unattended operation of $35 \mathrm{KV}$ substations.

\section{Inspection Robot System}

The inspection robot system for $35 \mathrm{KV}$ substations includes mobile robot and client monitoring software. The robot (Fig.1) is seated in substations while monitoring software is installed on the 
computer in surveillance room at the power company. The robot and computers can realize two-way communication through the internet.

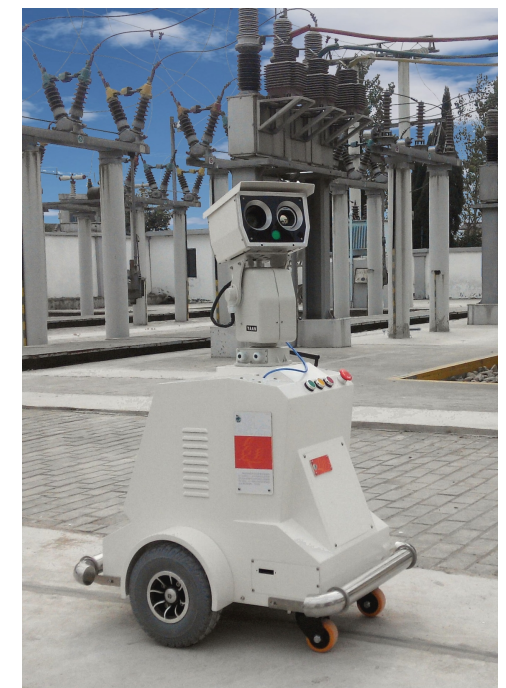

Fig 1 Inspection robot

\section{Overall Structure}

In consideration of information security, State Grid physically separates local area network (LAN) from wide area network (WAN) and prohibits the wireless access point. Therefore, inspection robot can only communicate through self communication network. In this paper, the inspection robot system communicates through Internet. Communication program is shown in Figure 2. Client monitoring software installed on the computer in the surveillance room of the power company, and the computer can connect to the Internet through a router. Meanwhile, there's a wireless router, which is connected to the Internet via dial-up, installed in $35 \mathrm{KV}$ substations. The inspection robot is connected to the wireless router via a wireless network card of its inside industrial computer. The area of $35 \mathrm{KV}$ substations is small, thus a three antennas router in the station's surveillance room can achieve full Wi-Fi wireless coverage within the substation.

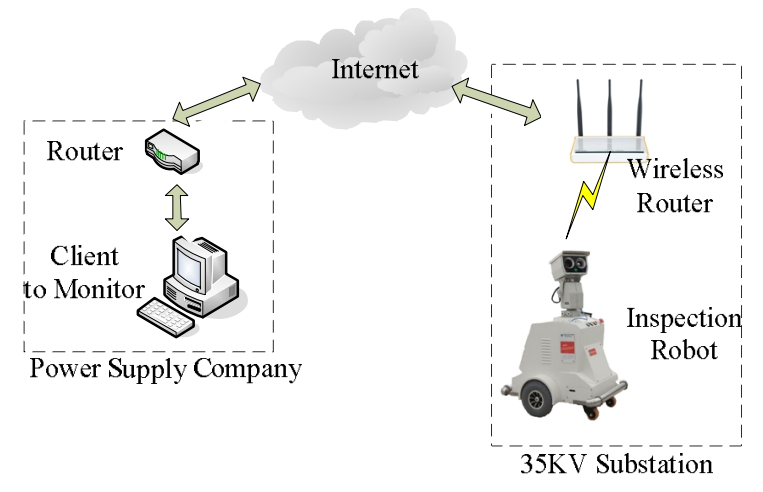

Fig 2 Communications schematic diagram

\section{Client monitoring software}

Point to point communications between Client monitoring software and inspection robot can be achieved by using the technology of Network Address Translation-Dynamic Domain Name Server (NAT-DDNS). In this paper, Peanut Hull software is used to realize NAT-DDNS technology. For the actual demand of $35 \mathrm{KV}$ unmanned substations, client monitoring software has two functions as motion control, monitoring and diagnosing. Client monitoring software is shown in Figure 3.

The motion control function includes start inspection, midway stopping, midway starting, one-key returning, controlling of the horizontal angle and pitch angle of PTZ. 
Monitoring diagnosis is that the software can receive and display real-time video sent by inspection robot through the Internet. The video includes visible and infrared thermal imaging. Monitor the substation equipments' appearance and temperature in real-time by using image recognition and contrast technologies at the same time.

For security consideration, the software has a password, only people who have authorities can use it.

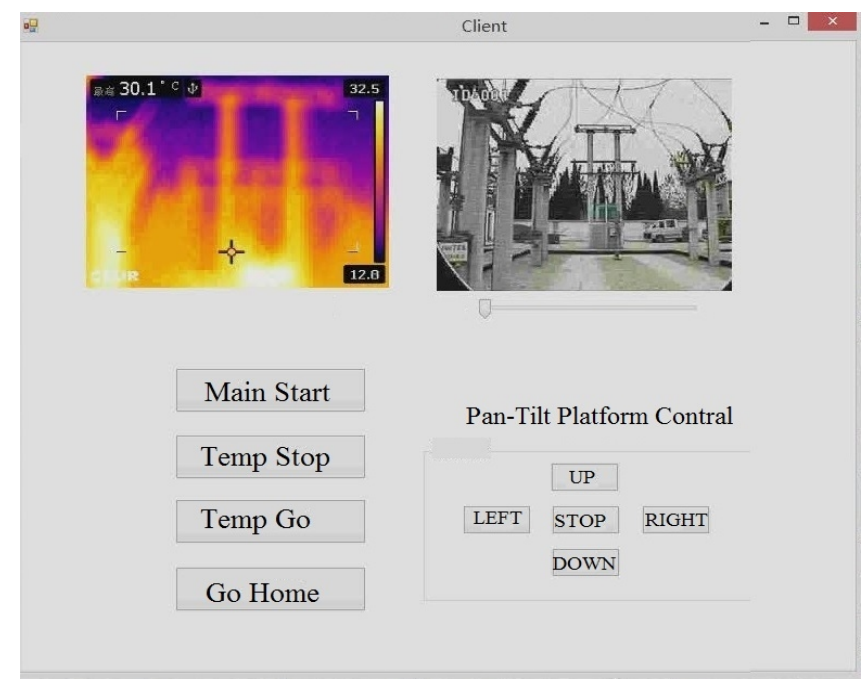

Fig 3 Client monitoring software

\section{The Control System of Inspection robot}

The control system structure of inspection robot is shown in Figure 4. The controller of the system has double forms as Industrial computer controller and microcontroller. Industrial computer controller has advantages as small size, high performance and strong capacity of anti-interference. And because the use of Windows XP operating system, Industrial computer controller provides convenience for programming of control software and remoting desktop maintenance of the inspection robot.Industrial computer controller is inspection robot's core of control. First, the industrial control computer obtains the image information collecting from HD cameras and an infrared infrared thermometer via USB video capture card.Then through the RS232 serial port to connect the RFID tag reader, servo motor and Head structure.Finally, the industrial control computer equipped with a wireless network card, whose antenna leads to the outside of the inspection robot's shell, to ensure a stable connection Wi-Fi wireless networks.

Microcontroller, as a secondary controller, is responsible for button detection, magnetic track position read, charging plug motor control and indicator light control. SCM uses a custom protocol to communicate with Industrial computer controller through RS232 serial port.

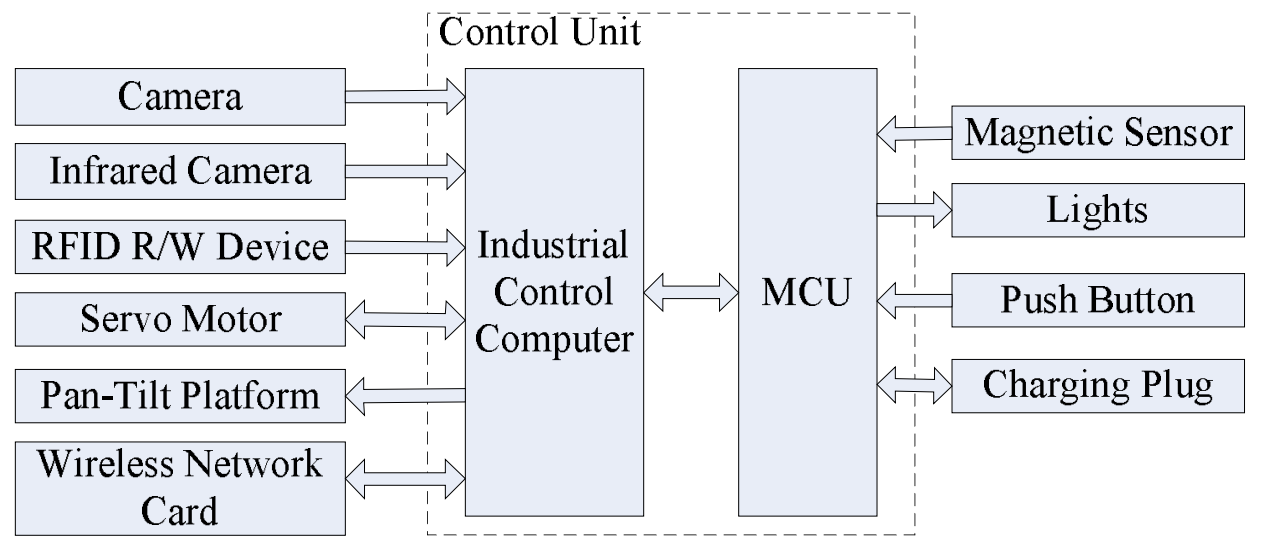

Fig 4 Block diagram of the control system 


\section{WCF-based Internet-point communication}

WCF (Windows Communication Foundation) is a series of application framework developed by Microsoft for supporting data communications. The framework is the best practices that developing distributed applications on the Windows platform.

Client monitoring software and inspection robots realize two-way communicate via WCF framework. Its communications using conventional Client (client) / Server (server) architecture. In the inspection robot system, the client monitoring software is Client side, and inspection robot is the Server side. A contract set the communication mode of the communicating parties. The communication method of communicating parties to follow is set by the protocol bindings. The security during communication is set by the communicating parties agreed safety levels.

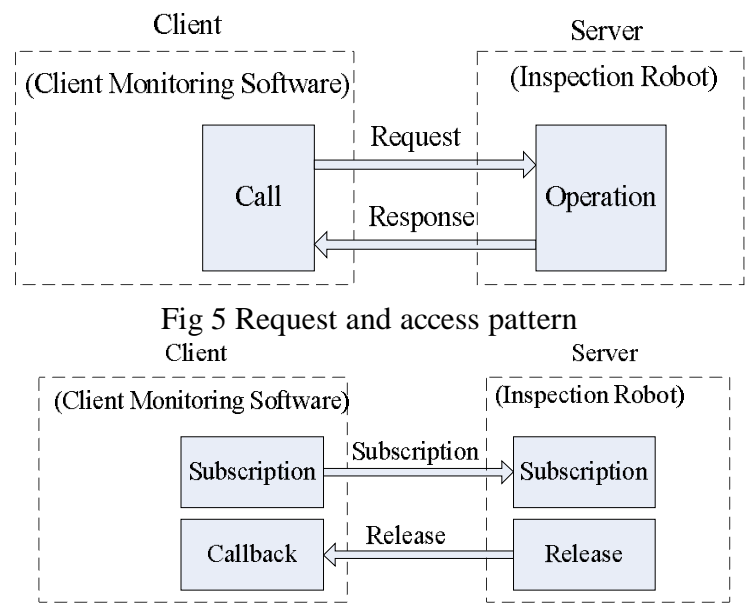

Fig 6 Send and subscription pattern

The control commands (such as start inspection robot, halfway temporary stop, etc.),sent by client monitoring software, use the WCF request and access mode interacting (its interaction diagram shown in Figure 5). Client side sends a request to the Server side, Server side completes control request based on command. Via WCF subscription model pushing the video stream to Client side,get video images(its interaction diagram shown in Figure 6).

When you start the Client-Side will be registered in the Server-side. Server-side saves Client-Side callback channel in memory references. When the video stream refresh, Server-side will push video stream in the form of a binary array to Client-side via saved references.

\section{Appearance monitoring and temperature monitoring}

The inspection robot on standby 24 hours a day. Transport inspection personnel remotely start scanning task through the client monitoring software in the control room. At this point inspection robot automatically goes away from the charging point and uses magnetic track navigation mode to perform inspection tasks. This paper to identify different substation equipment via RFID (radio frequency identification). Inspection robot use the equipped HD cameras and infrared thermometer to shoot an external view and infrared thermal image of a specific device. Through these images Staff detect thermal defects and abnormal appearance of the equipment (equipment damage, foreign matter adhesion, etc.)timely. In addition, inspection robot real-time upload visible and infrared thermal imaging video to the client monitoring software in the inspection process for transport inspection personnel analysis. and substation equipment appearance and infrared thermal image pattern will be captured in a specific naming deposit into the robot internal hard disk. 

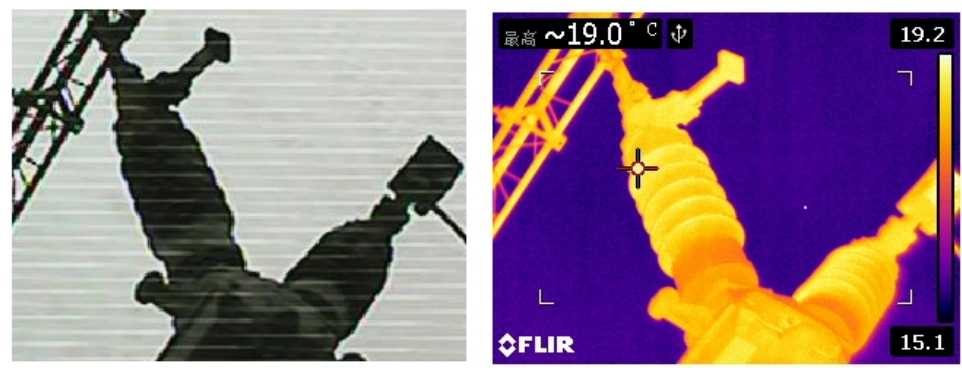

Fig7 Figure of breaker's appearance and infrared imaging

Appearance of a knife and infrared thermal shot by inspection robot as shown in Figure 7. The industrial computer controller gets the current infrared image frame maximum temperature. And check the image similarity of current visible and the standard photo Photo Gallery photo. Once the maximum temperature exceeds the set threshold value, or the difference between current photo and the standard photo library visible in the photo is greater than the allowable range of procedures, inspection robot will immediately send an alert message to the client monitoring software, notifying transport inspection staff dealt with accordingly.

\section{Automatic charging system}

Most of the current common charging systems for substation inspection robot use side docking mode. Due to the presence of the robots magnetic navigation errors, there will be occasional butt unsuccessful phenomenon. To solve this problem, we design new charging mechanism (see Figure 8). Charging socket mounted on the charging compartment floor (see Figure 9). It is equipped with a guide groove. Charging plug is installed on the inspection robot chassis (Figure 9). Charging plug is made of nylon, which is mounted to the top conductive copper plug. Charging plug 's tip structure is beveled, so that can achieve mechanical correction of the charging socket by means of the guide grooves when emerge the magnetic navigation deviation. Charging plug achieves the stretching and contraction of the plug by pusher motor.

Figure 10 shows the automatic charging docking procedure:

Step 1: inspection robot enters the charging area, charging plug from the telescopic state to state half done.

Step 2: inspection robot rely magnetic track navigation and mechanical corrective action of charging socket slowly into charging receptacle.

Step 3: charging plug and charging shutter contact socket, in which case the charging plug is located directly above the charging socket, inspection robot to stop traveling.

Step 4: Charge plug from the half stretched state to the fully extended state, charging plug into the charging socket, complete charging docking.

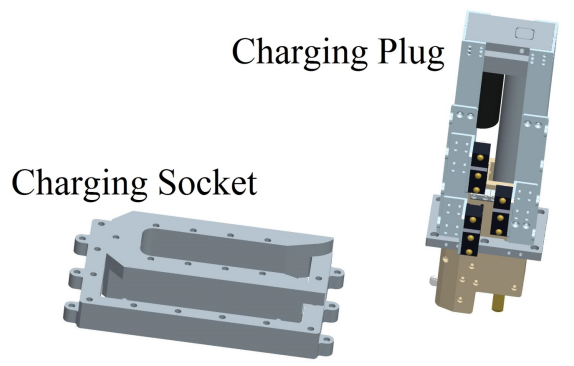

Fig 8 Automatic charging mechanism
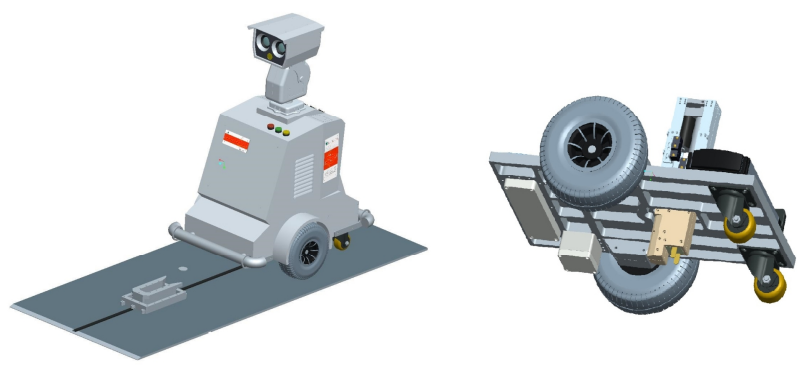

Fig 9 Assembly location of charging socket and charging plug 


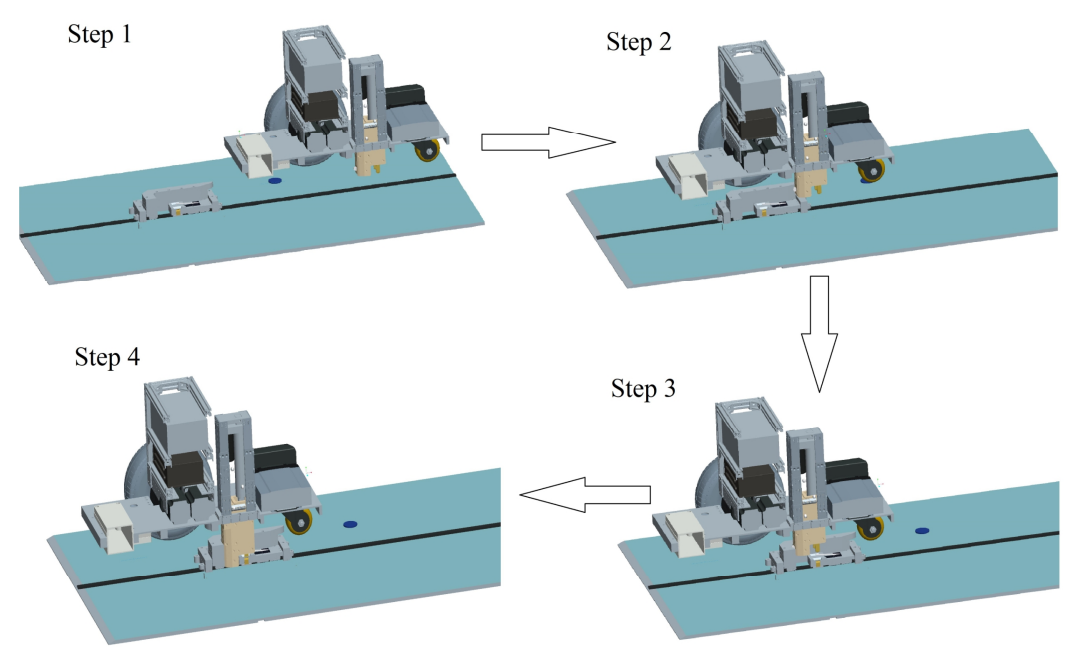

Fig 10 Docking process of automatic charging

\section{Conclusion}

Via the actual operation of a 35KV substation in Huainan City of Anhui Province shows the paper designed for small $35 \mathrm{KV}$ substation inspection robot running stability. Client software can monitor real-time control inspection robot motion. Inspection robot in the inspection process can automatically detect the appearance and temperature conditions of substation equipment. When Inspection robot find substation equipment abnormal appearance or abnormal temperature, it is possible to send alarm information in a timely manner to the client monitoring software for analysis. After the task is finished executing inspection robot can automatically return to the charging chamber and charging. Practice has proved that the design of substation inspection robot presentrd by this paper is feasible. The program effectively reduces the transport inspection staff labor intensity and improve their work efficiency.

\section{reference}

[1] Lu Shouying, Qian Qinglin, Zhang Bin, Wang Mingrui, Li Xiangdong, Wang Hong.Substation equipment inspection robot [J] Electric Power Systems, 2006,13: 94-98.

[2] Zhou Lihui, Zhang Yongsheng, Sun Yong, Liang Tao, Lu Shouying. Intelligent substation inspection robot development and application [J] Electric Power Systems, 2011,19: 85-88 + 96.

[3] Chen Qijuan, Mao Huihe, Xiao Zhihuai, Yu Bo, Liu Bin. Portable power equipment inspection apparatus [J] Electric Power Systems, 2001,03: 61-63.

[4] Zhang Xiping. Experience in building substation remote video surveillance system [J] Electric Power Systems, 2005,16: 97-99.

[5] Luo Junhao, Yan Yuwei. Substation automatic inspection robot system and its key technologies [J] Electrical Applications, 2014,13: 80-84. 